\title{
Lemur Tyrosine Kinase-3 Suppresses Growth of Prostate Cancer Via the AKT and MAPK Signaling Pathways
}

\author{
Pengcheng Sun ${ }^{a} \quad$ Xinhai Sun ${ }^{b}$ Weiming Zhao ${ }^{c}$ Minghua Ren ${ }^{c}$ Cheng Zhang ${ }^{c}$ \\ Ziqi Wang ${ }^{\mathrm{a}}$ Wanhai $\mathrm{Xu}^{\mathrm{a}}$ \\ aDepartment of Urology, the Fourth Hospital of Harbin Medical University, Harbin, ${ }^{b}$ Department of \\ general surgery, the Fourth Hospital of Harbin Medical University, Harbin, 'Department of Urology, the \\ First Hospital of Harbin Medical University, Harbin, China
}

\section{Key Words}

Prostate cancer $\bullet$ LMTK-3 $\cdot$ AKT $・$ MAPK $・$ Apoptosis

\begin{abstract}
Background/Aims: Lemur tyrosine kinase (LMTK)-3 is a member of the receptor tyrosine kinase (RTK) family. Abnormal expression of LMTK-3 exists in various types of cancers, especially in endocrine-resistant breast cancers; however, the precise level of expression and the biological function in prostate cancer are poorly understood. Methods: In the present study, we determined the expression of LMTK-3 in prostate cancer using immunohistochemistry and Western blotting. We infected PC3 and LNCaP cells with lentivirus-LMTK-3 and observed the biologic characteristics of the PC3 and LNCaP cells in vitro with TUNEL, and migration and invasion assays, respectively. We also established a transplant tumor model of human prostate cancer with infected cells in 15 BALB/c-nu/nu nude mice. Results: LMTK-3 was expressed in prostate epithelial cells. There was a significant decline in the level of LMTK-3 expression in prostate cancers compared to normal tissues. LMTK-3 inhibited PC3 and LNCaP cell growth, migration, and invasion, and induced cell apoptosis in vitro. We also observed that LMTK-3 induced PC3 cell apoptosis in vivo. Further study showed that LMTK-3 inhibited phosphorylation of AKT and ERK, and promoted phosphorylation and activation of p38 kinase and Jun kinase (JNK). Conclusion: Recombinant lentivirus with enhanced expression of LMTK3 inhibited prostate cancer cell growth and induced apoptosis in vitro and in vivo. AKT and MAPK signaling pathways may contribute to the process.
\end{abstract}

\section{Introduction}

Prostate cancer (PCa) is the second most common cancer in men [1]. Over the last 10 years, the incidence of PCa has increased in most countries and PCa represents a major disease burden worldwide [2,3]. Invasion and metastasis are crucial for the progression and

Wanhai Xu

KARGER 
prognosis of cancer [4]. Androgen, and the androgen receptor (AR) play a critical role in the development and progression of PCa and are the main targets for therapy; however, almost all patients develop castration-resistant PCa after a period of treatment [5-7]. Thus, new targets and treatment strategies are needed for PCa therapy.

Lemur tyrosine kinase (LMTK)-3 belongs to the serine-threonine-tyrosine kinases family. Studies have shown that abnormal expression of LMTK-3 is related to a number of malignant tumor types. LMTK-3 is significantly increased in human non-small cell lung cancer, and can be used as a biomarker to screen for primary non-small cell lung cancer and predict progression [8]. Lenz et al [9]. reported that LMTK-3 polymorphisms serve as a candidate prognostic factor for gastric cancer. Jiang et al [10]. confirmed that serum LMTK3 is a valuable biomarker for predicting the progression and prognosis of patients with colorectal cancer. Giamas et al. showed that high LMTK-3 is associated with proliferation of breast cancer, and silencing LMTK-3 reduces tumor volume [11]. LMTK-3 also contributes to endocrine resistance through estrogen receptor (ER)- $\alpha$ activity [12], however, the expression of LMTK-3 in PCa and the effect on PCa are still obscure.

In the current study we demonstrated that LMTK-3 is present in prostate epithelial cells. The expression of LMTK-3 was lower in PCa tissues compared with adjacent non-cancer tissues. We further explored the effect of LMTK-3 on PCa in vitro and in vivo, and the possible underlying mechanism.

\section{Materials and Methods}

\section{Patients and tissue samples}

Thirty-two paired PCa and adjacent normal prostate tissue samples were obtained from patients who underwent radical prostatectomies in the Department of Urology of the Fourth Affiliated Hospital of Harbin Medicine University (Harbin Heilongjiang, China). The tissues were separated and stored in liquid nitrogen until used. The 32 patients did not receive chemotherapy or radiotherapy and gave informed consent to participate in the study. Approval of the Ethics Committee of the Fourth Affiliated Hospital of Harbin Medical University was obtained. The clinicopathologic characteristics of the patients are presented in Table 1.

\section{Immunohistochemical staining}

The tissues from 32 patients were fixed in $10 \%$ neutral formalin, then embedded in paraffin. Immunohistochemistry staining was performed on $4-\mu \mathrm{m}$ paraffin sections. Briefly, paraffin sections were dewaxed with gradient alcohol and endogenous peroxidase was blocked for $20 \mathrm{~min}$ in $0.3 \%$ hydrogen peroxide in water. For pressure cooker antigen repair, the slides were treated in citrate buffer at $121^{\circ} \mathrm{C}$ for $5 \mathrm{~min}$. After cooling to room temperature, sections were incubated with rabbit antiLMTK-3 antibodies (1:500 dilution, NBP1-82887; Novus Biologicals, Littleton, CO, USA) at $4{ }^{\circ} \mathrm{C}$, thrice-washed with phosphate-buffered saline, then incubated with rabbit secondary antibodies for $20 \mathrm{~min}$ inside a humidor at room temperature. LMTK-3 expression was visualized with 3, 3-diaminobenzidine tetrahydrochloride, counterstained with Mayer's hematoxylin, dehydrated in gradient alcohol, sealed with neutral resin, and kept in the oven at $60^{\circ} \mathrm{C}$ for $48 \mathrm{~h}$.
Table 1. The clinicopathologic characteristics of the patients

\begin{tabular}{lc}
\hline Variable & Patient \\
\hline Age(years) & \\
Mean(range) & $72.6(33)$ \\
Min & 56 \\
Max & 89
\end{tabular}

PSA level, $\mathrm{ng} / \mathrm{mL}$

Mean(range)

29.5(96)

$\operatorname{Min}$

4

Max

100

Bone metastases, n (\%)

Present

18(56.25)

Absent

14(43.75)

Gleason Score, n (\%)

$\leq 7$

11(34.4)

8-10

21(65.6) 


\section{Cellular Physiology Cell Physiol Biochem 2017;42:2582-2592 \begin{tabular}{ll|l} 
and Biochemistry Published onlIne: August 24, 2017 & $\begin{array}{l}\text { (c) } 2017 \text { The Author(s). Published by S. Karger AG, Basel } \\
\text { www.karger.com/cpb }\end{array}$
\end{tabular}}

Sun et al.: LMTK3 Suppresses Prostate Cancer

\section{Cell cultures}

PC3 and LNCaP cells were obtained from the Cell Bank of the Chinese Academy of Science (Shanghai, China) and cultured in RPMI-1640 medium supplemented with 10\% heated-inactivated fetal bovine serum (FBS; Gibco, New York, NY,,USA). Incubations were carried out at $37{ }^{\circ} \mathrm{C}$ in a humidified atmosphere of $5 \%$ $\mathrm{CO}_{2}$. Cells in the logarithmic growth phase were used for further experiments.

\section{Lentivirus infection and transfection of siRNA}

Plasmid vector pLenti6.3-LMTK3-IRES-EGFP and control recombinant lentivirus (Lv-LMTK3 and Lv were constructed by the Genechem Company (Shanghai, China). A total of $1 \times 10^{5}$ cells were seeded in a 6-well cell plate and incubated for $12 \mathrm{~h}$ to reach 30\% confluence, then infected with Lv-LMTK3 (LMTK3 overexpression group), Lv (negative control group), and no infection (non-transfected control group) at a multiplicity of infection (MOI) of 20 transducing units (TUs) per cell. After infection for 4 h, virusfree medium with 10\% FBS was changed to culture the cells. Three days later, the efficiency of infection was generally determined by observing the expression of GFP density contained by lentivirus. Then, the cells were harvested for real-time quantitative polymerase chain reaction analysis. siRNA (50 nM) and its negative controls (Gene Pharma, Shanghai, China) were transfected using Lipofectamine 2000 (Invitrogen, Carlsbad, CA, USA) in serum-free Opti-MEM (Invitrogen) according to the manufacturer's instructions. Cells were collected $48 \mathrm{~h}$ later. Transfection efficiency was confirmed by real-time PCR. All siRNA sequences are listed in Table 2.

\section{Animals and heterotopic tumor growth assay}

Four-week-old male BALB/c-nu/nu mice were obtained from The Laboratory Animal Center of Shanghai. All animal procedures were approved by the Institutional Animal Care and Use Committee at the Fourth Hospital Affiliated to Harbin Medical University. The nude mice were randomly assigned into 3 groups $(n=5)$. To establish the xenografts, $1 \times 10^{6}$ infected PC3 cells were injected subcutaneously in nude mice with Matrigel (BD Bioscience, San Jose, CA, USA). Animals were carefully monitored, and tumor volume was measured by caliper measurement every 2 days and calculated according to the following formula: volume=length $\times$ width ${ }^{2}$ /2. The curve of the tumor volume was drawn. Two weeks after implantation, the mice were sacrificed and the xenografts were further analyzed through Western blot, real-time quantitative polymerase chain reaction analysis, and a TUNEL assay.

Cell viability assay

The MTT assay was used to analyze the viability of PC3 and LNCaP cells after treatment with Lv-LMTK3 and siRNA. The cells were seeded separately at an equivalent density of $2 \times 10^{3}$ cells/well in 96 -well plates and cultured at $37^{\circ} \mathrm{C}$ for 4 days. Then, $10 \mu \mathrm{l}(5 \mathrm{mg} / \mathrm{ml})$ of MTT (Sigma, St. Louis, MO, USA) was added to each well. After a 4-h incubation with MTT at $37^{\circ} \mathrm{C}$, the supernatant was removed and $150 \mu \mathrm{l}$ of dimethyl sulfoxide (DMSO; Sigma) was added to each well to dissolve the crystal. Ten minutes later, the absorbance was measured at $490 \mathrm{~nm}$ with a microplate reader. Each assay was performed in triplicate.

\section{Migration and invasion assays}

To determine the effect of LMTK3 on cell migration, the Transwell assay was carried out. Briefly, infected and non-infected cells were suspended $\left(1 \times 10^{5} /\right.$ well) in the upper chambers (Corning, Cambridge, MA. USA) containing $200 \mu \mathrm{L}$ of serum-free RPMI-1640 medium. Medium containing 10\% FBS was then added to the lower chamber as a chemoattractant. After incubation for $24 \mathrm{~h}$, the non-migrating cells on the polycarbonate membranes were wiped off using a cotton swab. Then, the polycarbonate membranes were handled with $4 \%$ paraformaldehyde for $30 \mathrm{~min}$ and stained with $0.2 \%$ crystal violet for $20 \mathrm{~min}$. The number of cells that had crossed through the polycarbonate membranes was recorded and analyzed. Matrigel-precoated Transwell inserts (BD Bioscience, San Jose, CA, USA) were used for the cell invasion assay. The membrane at the bottom of the Transwell chamber was coated with a 1:8 Matrigel suspension, followed by a 1-h incubation at room temperature. Similarly, the lower chamber was filled with $10 \%$ FBS 


\section{Cellular Physiology Cell Physiol Biochem 2017;42:2582-2592 \begin{tabular}{l|l|l} 
and Biochemistry Published onlıne: August 24, 2017 & $\begin{array}{l}\text { (c) } 2017 \text { The Author(s). Published by S. Karger AG, Basel } \\
\text { www.karger.com/cpb }\end{array}$
\end{tabular} \\ Sun et al.: LMTK3 Suppresses Prostate Cancer}

as a chemoattractant. After $24 \mathrm{~h}$ of incubation at $37^{\circ} \mathrm{C}$ in $5 \% \mathrm{CO}_{2}$, the upper layer of cells on the membrane were removed, and the cells that had invaded through the membrane were fixed, stained, and counted. Each experiment was performed in triplicate. Three randomly selected fields were counted for the number of invaded cells. The number of invaded cells $=$ (number of cells in all three fields) $/ 3$.

TUNEL assays

Apoptosis was evaluated using the TUNEL assay. The pre-treated PC3 and LNCaP cells $\left(1 \times 10^{4}\right)$ were seeded on coverslips in 6-well plates and cultured for 3 days. Then, the coverslips were fixed, permeabilized, and stained using an In Situ Cell Death Detection Kit (Roche, Mannheim, Germany) according to the manufacturer's instructions. Portions of the xenografts were fixed with $10 \%$ paraformaldehyde. Sixmicrometer thick frozen sections were also detected by staining xenografts with the In Situ Cell Death Detection Kit, according to the manufacturer's instructions. After TUNEL staining, the frozen sections were immerged into DAPI (Invitrogen) solution to stain nuclei. Fluorescence staining was viewed by a laser scanning confocal microscope (FV300; Olympus, Tokyo Japan). Each stained section was examined under a high power field (magnification, $\times 400$ ).

\section{Quantitative real-time polymerase chain reaction ( $q R T-P C R$ )}

Total RNA was extracted using Trizol reagent (Invitrogen) from PCa tissues, adjacent normal prostate tissues, PC3 cells, LNCaP cells, and xenografts. For determination of LMTK3 expression, qRT-PCR was performed using SYBR Green Real-time PCR Master Mix (Takara, , Japan) according to the manufacturer's protocol. Relative expression was normalized to the expression of U6 small RNA and evaluated using SDS2.4 software (Applied Biosystems, Foster City, CA,USA) . The primers are listed in Table 2.

\section{Western blot}

The protein samples were extracted from the paired tissue samples, two PCa cells, and the xenografts. After quantification, proteins were separated by SDS-PAGE and transferred to a nitrocellulose membrane (Bio-Rad, Hercules, CA USA). After blocking with 5\% non-fat milk in Tris-buffered saline with Tween (TBST) for $2 \mathrm{~h}$, the membrane was incubated with the following primary antibodies in 5\% milk TBST: LMTK-3 (1:1000 dilution; Santa Cruz Biotechnology, Inc., Santa Cruz, CA, USA); Bcl-2 (1:1000 dilution; Cell Signaling Technology, Beverly, MA; USA); Bax (1:1000 dilution; Cell Signaling Technology); caspase-3 (1:500 dilution; Cell Signaling Technology); total Akt (1:200 dilution; Santa Cruz Biotechnology, Inc.); p-Akt (1:1000 dilution; Cell Signaling Technology); p-38 (1:800 dilution; Santa Cruz Biotechnology, Inc.); p-p38 (1:800 dilution; Santa Cruz Biotechnology, Inc.); ERK (1:500 dilution; Cell Signaling Technology); p-ERK (1:500 dilution; Cell Signaling Technology); JNK (1:800 dilution; Santa Cruz Biotechnology, Inc.); p-JNK (1:800 dilution; Santa Cruz Biotechnology, Inc.); and GAPDH (1:200 dilution; Santa Cruz Biotechnology, Inc.). Membranes were washed 3 times with PBS containing 0.5\% Tween 20 (PBS-T) and incubated with a secondary antibody (1:8000; Alexa Fluor ${ }^{\circledR} 488$ goat anti-mouse IgG [H+L] or Alexa Fluor ${ }^{\circ} 488$ goat anti-rabbit IgG [H+L], Invitrogen) at room temperature for $1.5 \mathrm{~h}$. Western blot bands were quantified using Odyssey v1.2 software (LI-COR Biosciences, Lincoln, NE, USA) by measuring the band intensity (area $\times$ optical density [OD]) for each group and normalized to GAPDH. All the experiments were repeated four times.

\section{Statistical analysis}

The data are expressed as the mean \pm SEM. All analyses were performed using GraphPad Prism (version 5.0) and SPSS 14.0. Two-tailed values of $\mathrm{P}<0.05$ were considered statistically significant with Student's t-test.

\section{Results}

The localization and expression of LMTK3 protein in prostate tissues

The aberrant expression of LMTK-3 has been observed in several human malignancie s [8-12]; however, the localization and level of LMTK-3 expression in prostate tissues is still unknown. In the current study, immunohistochemical staining was used to locate the LMTK-3 protein. As shown in Fig. 1, LMTK-3 was expressed in prostate epithelium cells, but minimally 
expressed in PCa cells. We used qRTPCR and Western blot to examine LMTK-3 expression profiles between PCa tissues and paired adjacent noncancerous prostate tissues from 32 individual patients. As shown in Fig. 2A and 2B, the level of LMTK-3 expression was significantly lower in the tumor specimens compared with normal tissues.

Overexpression of LMTK-3 decreased the viability of PC3 and $L N$ CaP cells in vitro

To investigate the influence of LMTK-3 on the biologic behavior of PCa cells, we infected PC3 and LNCaP cells with recombinant LV-LMTK-3 and determined cell viability. At the same time, we knocked down LMTK3 with siRNA in the two cell lines and examined cell viability. qRT-PCR was performed to guarantee the efficiency of transfection. As shown in Fig. 3A and $3 \mathrm{C}$, the expression of LMTK-3 was significantly increased when the two cell lines transfected with Lv-LMTK3 were compared with the control and Lv groups. The level of LMTK-3 expression decreased when using siRNA. The MTT assay showed that overexpression of LMTK-3 significantly decreased the viability of PC3 and LNCaP cells compared with the control and $\mathrm{LV}$ groups (Fig. 3B and 3D, p $\mathrm{P}<0.01$ ); however, there was no obvious difference after LMTK-3 knockdown with siRNA.

LMTK-3 attenuated the migration and invasion potential of PC3 and LNCaP cells in vitro

The invasion and migration ability of tumor cells is crucial for tumor progression. To observe the effect of LMTK-3 on cell migration and invasion, the Transwell and Matrigel invasion assays were used, respectively. The migratory potential of the Lv-LMTK3-infected PC3 and LNCaP cells was significantly reduced (Fig. $4 \mathrm{~A}$ and C). Moreover, the Matrigel invasion assay showed that the invasion of PC3 and LNCaP cells was inhibited by LMTK-3 compared to the control group (Fig. 4 B and D).

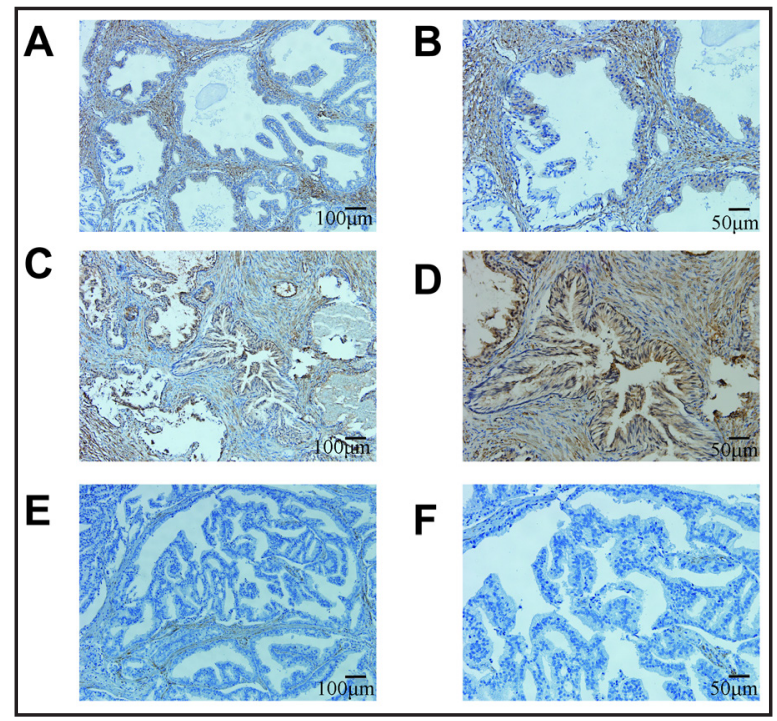

Fig. 1. Localization of LMTK-3 in prostate tissues. Immunohistochemistry was used to examine the expression of LMTK-3. A, B, C, and D showed that LMTK-3 was expressed in human prostate epithelium cells. E and F showed that LMTK-3 had minimal expression in human PCa tissues.

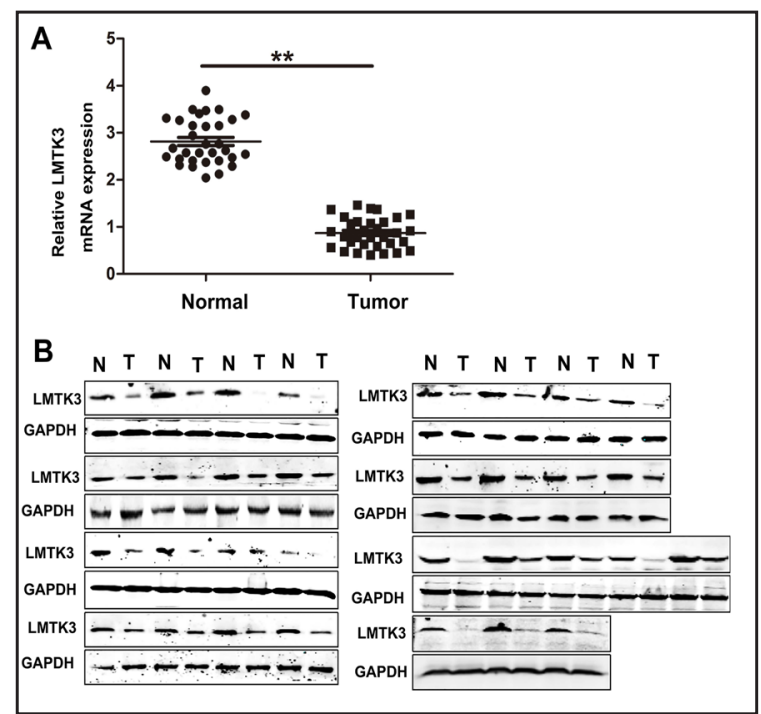

Fig. 2. Relative expression of LMTK-3 in PCa tissues. A and $\mathrm{B}$ showed the level of LMTK-3 mRNA and protein expression in normal and PCa tissues. LMTK-3 was down-regulated in PCa tissues compared to normal nontumor tissues. $\mathrm{n}=32$; ** represents $\mathrm{p}<0.05$.

\section{KARGER}


Fig. 3. Effects of LMTK-3 on cell viability and apoptosis. A and C showed the expression of LMTK3 in PC3 and LNCaP cells after infection with LvLMTK3 and knockdown of LMTK-3 with siRNA. The MTT assay was used to analyze the viability of PC3 and LNCaP cells after infection with Lv-LMTK3 or knockdown of LMTK-3 with siRNA (Fig. $3 \mathrm{~B}$ and $\mathrm{D}){ }^{* *}$ represents $\mathrm{p}<0.05$.
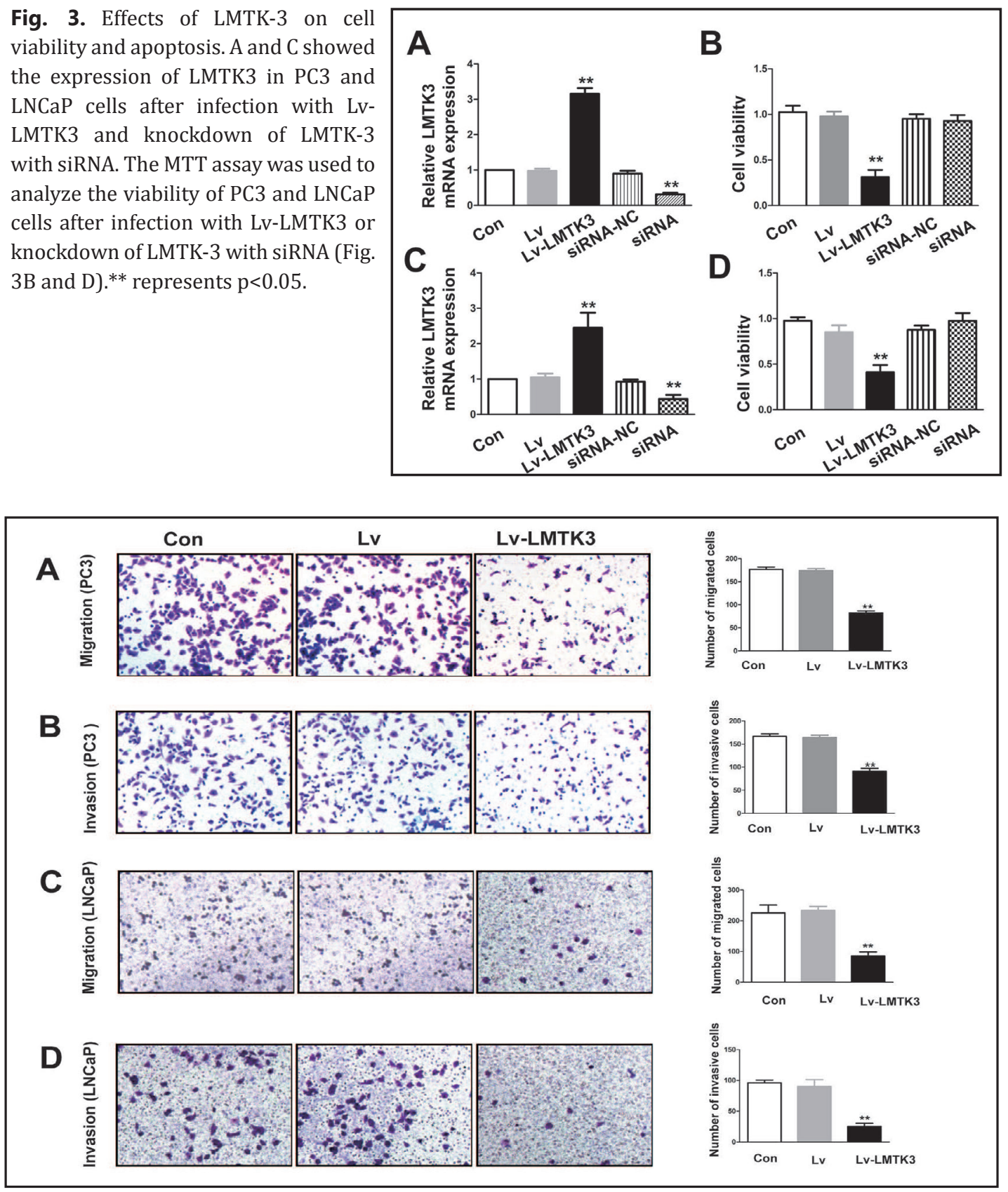

Fig. 4. Influence of LMTK-3 on migration and invasion potential of cells. Fig. 4A and 4C shows that LMTK-3 inhibited the migration of PC3 and LNCaP cells, respectively. Fig. 4B and 4D represented LMTK-3 repressed invasion of PC3 and LNCaP cells. ** represents $\mathrm{p}<0.05$.

Overexpression of LMTK-3 induced apoptosis of PC3 and LNCaP cells in vitro

To investigate whether or not LMTK-3 can induce PCa cell apoptosis, the TUNEL assay was used to examine cell apoptosis (Fig. 5A and B). We found that apoptosis was increased in PC3 and LNCaP cells after infection with Lv-LMTK3 in vitro (Fig. 5 C and D, $\mathrm{p}<0.05$ ). We also examined the expression of apoptosis-related protein, including Bax, Bcl-2, and caspase-3. As expected, the expression of Bax and caspase-3 was increased in PC3 cells (Fig. 5F and G, $\mathrm{p}<0.05$ ) and LNCaP cells (Fig. 5I and J, p <0.05). By contrast, the expression of Bcl-2 was decreased in PC3 and LNCaP cells (Fig. 5E and H, p $<0.05$ ). 


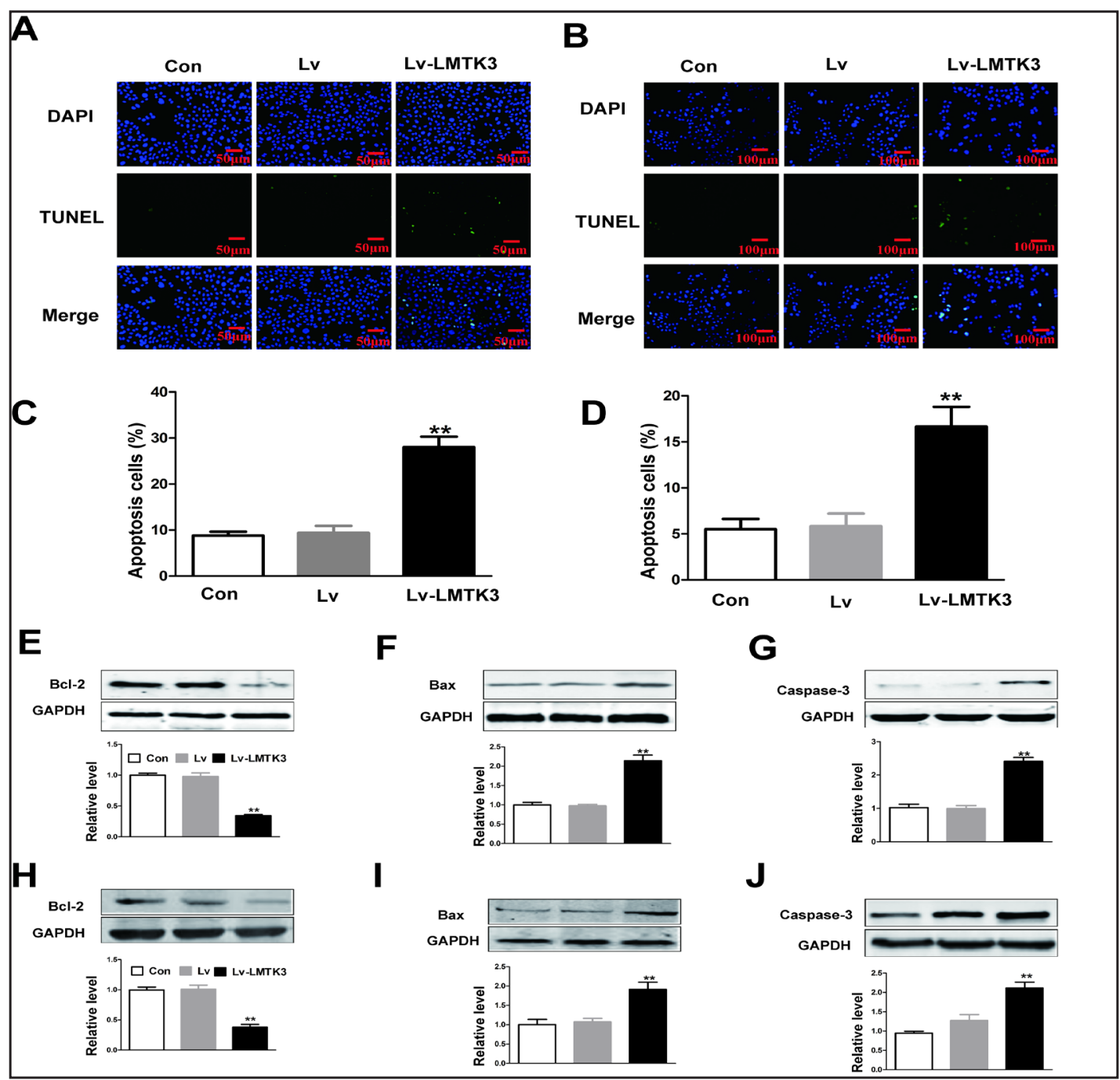

Fig. 5. LMTK3 induced PCa cell apoptosis. TUNEL assays and Western blot were used to demonstrate the change in apoptosis. A and C showed that LMTK-3 induced PC3 cell apoptosis. B and D showed LMTK-3 induced LNCaP cell apoptosis. E, F, and G showed the change in Bcl-2, Bax, and caspase-3 in PC3 cells after infection with- Lv-LMTK3. H, I, and J showed the change in Bcl--2, Bax, and caspase-3 in LNCaP cells after infection with Lv-LMTK3. ${ }^{* *}$ represents $\mathrm{p}<0.05$.

\section{LMTK-3 inhibited tumorigenicity of PC3 cells and induced apoptosis in vivo}

We constructed the subcutaneous tumor model with infected and uninfected PC3 cells to further verify the effect of LMTK-3 on tumor growth in vivo. An equal amount of PC3 cells was injected subcutaneously in nude mice. The expression of LMTK-3 in xenografts was much higher in the Lv-LMTK3 group than the control group at the mRNA and protein levels, suggesting that we successfully overexpressed LMTK-3 in vivo (Fig. 6A and 6B, p $<0.05$ ). The volume of tumors was significantly smaller in the Lv-LMTK-3 group compared with the control group (Fig. 6C and 6D, $\mathrm{p}<0.05$ ). To further confirm the mechanism underlying LMTK-3-induced tumorigenicity of PC3 cells in vivo, TUNEL staining was performed. We found that LMTK-3 increased the ratio of apoptosis cells in vivo (Fig. $6 \mathrm{E}$ and $6 \mathrm{~F}, \mathrm{p}<0.05$ ). At the same time, we sacrificed the mice and performed a proteomic analysis of tumor tissue, and found that the expression of apoptosis-related proteins changed. As shown in Fig. 6G, the expression of $\mathrm{Bcl}-2$ was decreased $(\mathrm{p}<0.05)$. Instead, the expression of Bax and caspase- 3 was increased (Fig. $6 \mathrm{H}$ and I, $\mathrm{p}<0.05$ ).

\section{KARGER}




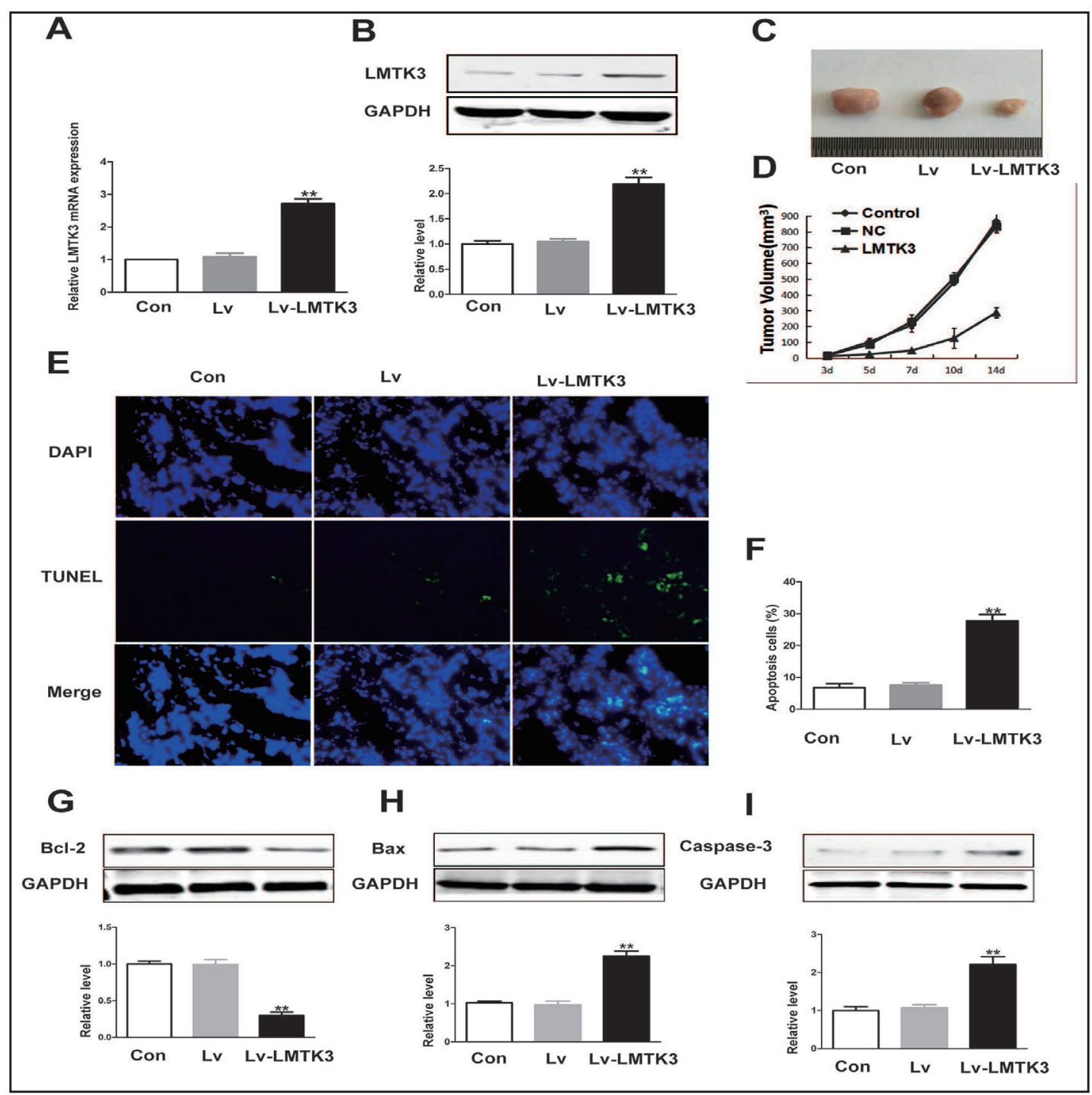

Fig. 6. The effects of LMTK-3 on tumorigenicity of PC3 cells in vivo. A and B showed the expression of LMTK3 in xenografts. $\mathrm{C}$ and $\mathrm{D}$ showed the tumor volume in different groups and the tumor growth curve. $\mathrm{E}$ and F showed cell apoptosis. G, H, and I showed the change in apoptosis-related proteins in the three groups of tumor tissues. ${ }^{* *}$ represents $\mathrm{p}<0.05$.

The PI3K/AKT and mitogen-activated protein kinase (MAPK) signaling pathways are involved in LMTK-3-induced apoptosis

To explore the possible mechanism underlying LMTK-3-induced apoptosis, we examined the expression of subfamilies of MAPKs. Western blot analysis showed that the expression of total AKT, P38, ERK, and JNK did not change; however, phosphorylated AKT and ERK1/2 were down-regulated after LMTK-3 treatment (Fig. 7A and 7C, p <0.05). In contrast, phosphorylated p38 and JNK were significantly increased (Fig. 7B and 7D, p <0.05). In addition, we detected the mechanism of LMTK3 induced apoptosis in vivo. Similar to the in vitro experiments, there were no significant differences in AKT, P-38, ERK, and JNK expression between the different groups of xenografts. P-AKT and P-ERK were downregulated, whereas the expression of P-P38 and P-JNK was up-regulated (Fig. 8A -D, p<0.05). In summary, the data demonstrated that the PI3K/AKT and MAPK signaling pathways may contribute to LMTK-3-induced apoptosis. 


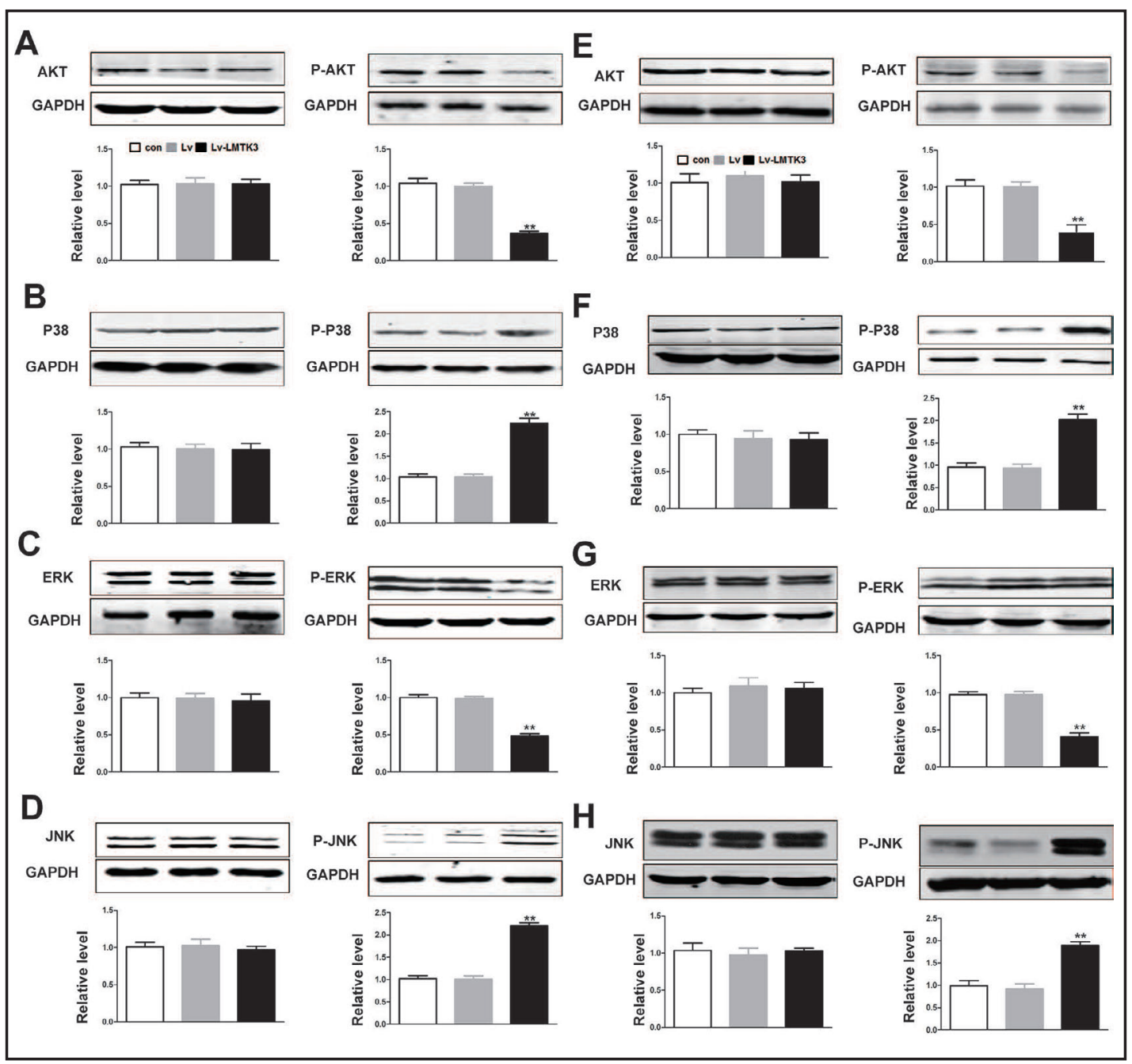

Fig. 7. Change in the MAPK signal pathway after infection of Lv-LMTK-3 in vitro. $A, B, C$, and $D$ showed the changes in AKT, p-AKT, P38MAPK, P-P38MAPK, ERK, P-ERK, JNK , and P-JNK in PC3 cells. E, F, G, and $\mathrm{H}$ showed the expression of corresponding proteins in LNCaP cells after infection with Lv-LMTK-3. ** represents $\mathrm{p}<0.05$.

\section{Discussion}

Studies showed that abnormal expression of LMTK-3 was identified in several types of cancers, including breast, non-small cell lung, colorectal, and gastric cancer, which may serve as a new reliable diagnostic and independent prognostic biomarker in these cancers [8-10]; however, the expression of LMTK-3 in PCa and the effect of LMTK-3 on PCa are still unclear. In the current study, we showed that the expression of LMTK-3 in PCa was decreased compared with normal prostate tissues, which suggested that LMTK-3 may act as a tumor suppressor in PCa.

To observe the influence of LMTK-3 on malignant biologic behavior, the PC3 and LNCaP cell lines were cultured and infected by lentiviurs to deliver LMTK-3. We showed that LMTK3 inhibited the viability and induced apoptosis of the two cell lines in vitro. The migration and invasion of PC3 and LNCaP cells were also inhibited. We also constructed a subcutaneous tumor model in nude mice to verify the effect of LMTK-3 on the growth of PCa cells in vivo, and found that LMTK-3 indeed inhibited tumor growth and decreased tumor volume. The apoptosis rate was also increased in vivo after infection with LMTK-3 lentivirus. We examined 
the expression of apoptosis-related proteins and showed that LMTK3 increased the level of $\mathrm{Bcl}-2$ protein, and attenuated the expression of Bax and caspase- 3 proteins in vitro and in vivo $[13,14]$. Thus, we concluded that the tumor suppression effect of LMTK3 in PCa was mediated by regulating cell apoptosis.

It is well-known that the three most important subfamilies of MAPKs (extracellular signal regulated kinases 1 and 2 [ERK1/2], c-Jun NH2-terminal kinase [JNK], and p38 mitogenactivated protein) play a key role in cell growth, differentiation, and apoptosis in response to a variety of stresses or stimuli, including cancers [15-17]. The phosphatidylinositol 3-kinase/protein kinase-B (PI3K/ AKT) cell signaling cascade is another important intracellular pathway, is frequently activated in diverse cancers, and regulates cell proliferation, cell differentiation, cellular apoptosis, and cancer cell survival $[18,19]$. Studies have shown that AKT activation is also involved in migration of $\mathrm{PCa}$ cells [20]. We examined activation of the AKT, p38MAPK, JNK, and ERK signaling pathways in PC3 cells and xenografts to explore the possible mechanism underlying the LMTK3 effect. We found that LMTK-3 increased phosphorylated p38 and JNK, but decreased phosphorylated ERK1/2 and AKT. These data indicate that the change in these signaling pathways after overexpression of LMTKs may contribute to biologic behavior changes of PCa, especially cell apoptosis.

\section{Conclusion}

In summary, we found that the expression of LMTK-3 was lower in PCa tissues than normal tissues. LMTK-3 induced PCa apoptosis in vitro and in vivo; the AKT and MAPK signaling pathways may contribute to the process. LMTK-3 may be a promising intervention target in the management of PCas.

\section{Acknowledgements}

This study was supported by the National Natural Science Foundation of China (No. 81270022).

\section{Disclosure Statement}

The authors declare that they have no conflicts of interest.

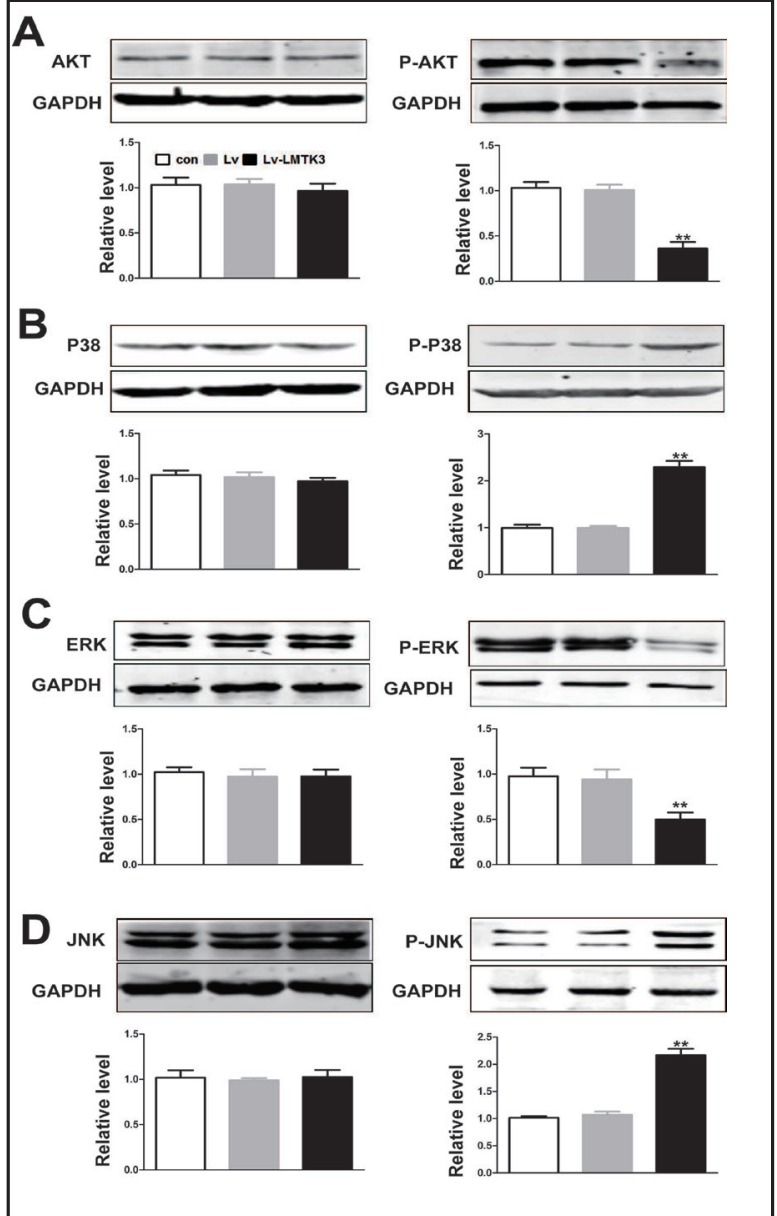

Fig. 8. Change in the MAPK signal pathway after infection with Lv-LMTK-3 in vivo. A, B, C and D showed the change of AKT, p-AKT, P38MAPK, P-P38MAPK, ERK, P-ERK, JNK, and P-JNK in xenografts by Lv-LMTK-3-infected PC3 cells ** represents $\mathrm{p}<0.05$. 


\section{Cellular Physiology Cell Physiol Biochem 2017;42:2582-2592 \begin{tabular}{ll|l} 
and Biochemistry Published onlıne: August 24, 2017 & $\begin{array}{l}\text { (c) } 2017 \text { The Author(s). Published by S. Karger AG, Basel } \\
\text { www.karger.com/cpb }\end{array}$ \\
\hline
\end{tabular}}

\section{References}

1 Ferlay J, Soerjomataram I, Dikshit R, Eser S, Mathers C, Rebelo M, Parkin DM, Forman D, Bray F: Cancer incidence and mortality worldwide: sources, methods and major patterns in GLOBOCAN 2012. Int J Cancer 2015;136:E359-386.

-2 Center MM, Jemal A, Lortet-Tieulent J, Ward E, Ferlay J, Brawley 0, Bray F: International variation in prostate cancer incidence and mortality rates. Eur Urol 2012;61:1079-1092.

-3 Huang S, He P, Peng X, Li J, Xu D, Tang Y: Pristimerin Inhibits Prostate Cancer Bone Metastasis by Targeting PC-3 Stem Cell Characteristics and VEGF-Induced Vasculogenesis of BM-EPCs. Cell Physiol Biochem 2015;37:253-268.

-4 Hu Q, Tong S, Zhao X, Ding W, Gou Y, Xu K, Sun C, Xia G: Periostin Mediates TGF-beta-Induced Epithelial Mesenchymal Transition in Prostate Cancer Cells. Cell Physiol Biochem 2015;36:799-809.

-5 Galsky MD, Small AC, Tsao CK, Oh WK: Clinical development of novel therapeutics for castration-resistant prostate cancer: historic challenges and recent successes. CA Cancer J Clin 2012;62:299-308.

6 Messing EM, Manola J, Sarosdy M, Wilding G, Crawford ED, Trump D: Immediate hormonal therapy compared with observation after radical prostatectomy and pelvic lymphadenectomy in men with nodepositive prostate cancer. N Engl J Med 1999;341:1781-1788.

7 Katzenwadel A, Wolf P: Androgen deprivation of prostate cancer: Leading to a therapeutic dead end. Cancer Lett 2015;367:12-17.

-8 Zhang K, Chen L, Deng H, Zou Y, Liu J, Shi H, Xu B, Lu M, Li C, Jiang J, Wang Z: Serum lemur tyrosine kinase-3: a novel biomarker for screening primary non-small cell lung cancer and predicting cancer progression. Int J Clin Exp Pathol 2015;8:629-635.

-9 Wakatsuki T, LaBonte MJ, Bohanes PO, Zhang W, Yang D, Azuma M, Barzi A, Ning Y, Loupakis F, Saadat S, Volz N, Stintzing S, El-Khoueiry R, Koizumi W, Watanabe M, Shah M, Stebbing J, Giamas G, Lenz HJ: Prognostic role of lemur tyrosine kinase-3 germline polymorphisms in adjuvant gastric cancer in Japan and the United States. Mol Cancer Ther 2013;12:2261-2272.

10 Shi H, Wu J, Ji M, Zhou Q, Li Z, Zheng X, Xu B, Deng H, Zhao W, Wu C, Jiang J: Serum lemur tyrosine kinase 3 expression in colorectal cancer patients predicts cancer progression and prognosis. Med Oncol 2013;30:754.

11 Giamas G, Filipovic A, Jacob J, Messier W, Zhang H, Yang D, Zhang W, Shifa BA, Photiou A, Tralau-Stewart C, Castellano L, Green AR, Coombes RC, Ellis IO, Ali S, Lenz HJ, Stebbing J: Kinome screening for regulators of the estrogen receptor identifies LMTK3 as a new therapeutic target in breast cancer. Nat Med 2011;17:715719.

12 Stebbing J, Filipovic A, Ellis IO, Green AR, D’Silva TR, Lenz HJ, Coombes RC, Wang T, Lee SC, Giamas G: LMTK3 expression in breast cancer: association with tumor phenotype and clinical outcome. Breast Cancer Res Treat 2012;132:537-544.

13 Yip KW, Reed JC: Bcl-2 family proteins and cancer. Oncogene 2008;27:6398-6406.

-14 Li P, Nijhawan D, Budihardjo I, Srinivasula SM, Ahmad M, Alnemri ES, Wang X: Cytochrome c and dATP-dependent formation of Apaf-1/caspase-9 complex initiates an apoptotic protease cascade. Cell 1997;91:479-489.

15 Chun JS: Expression, activity, and regulation of MAP kinases in cultured chondrocytes. Methods Mol Med 2004;100:291-306.

-16 Farooq A, Zhou MM: Structure and regulation of MAPK phosphatases. Cell Signal 2004;16:769-779.

17 Cross TG, Scheel-Toellner D, Henriquez NV, Deacon E, Salmon M, Lord JM: Serine/threonine protein kinases and apoptosis. Exp Cell Res 2000;256:34-41.

18 Fresno Vara JA, Casado E, de Castro J, Cejas P, Belda-Iniesta C, Gonzalez-Baron M: PI3K/Akt signalling pathway and cancer. Cancer Treat Rev 2004;30:193-204.

19 Engelman JA: Targeting PI3K signalling in cancer: opportunities, challenges and limitations. Nat Rev Cancer 2009;9:550-562.

20 Young B, Purcell C, Kuang YQ, Charette N, Dupre DJ: Superoxide Dismutase 1 Regulation of CXCR4Mediated Signaling in Prostate Cancer Cells is Dependent on Cellular Oxidative State. Cell Physiol Biochem 2015;37:2071-2084. 